\title{
Emergent Spatial Patterns in Vegetable Population Dynamics: Towards Pattern Detection and Interpretation
}

\author{
Stefania Bandini, Sara Manzoni, Stefano Redaelli, and Leonardo Vanneschi \\ Dept. of Informatics, Systems, and Communication \\ University of Milan-Bicocca \\ Via Bicocca degli Arcimboldi 8, 20126 Milan, Italy \\ \{bandini, manzoni, redaelli, vanneschi\}@disco.unimib.it
}

\begin{abstract}
In this paper we present an ongoing research that aims at providing an interpretation and detection method for spatial patterns supporting ecosystem management in the study of forest systems according to a distributed modeling and simulation approach. To this aim an innovative analysis method inspired by the Chinese Go game is under design. The originality of the approach concerns the detection within system configurations of known patterns whose interpretations are wellknown by expert Go players.
\end{abstract}

\section{Introduction}

The CAFFE (Cellular Automata For Forest Ecosystems) project is an interdisciplinary research that involves computer science, biology, and ecosystem management. It involves the Artificial Intelligence Lab (L.INT.AR.) of the Department of Informatics, Systems and Communication of the University of Milano-Bicocca and the System Research Department of Austrian Research Center (ARC). The main aim of this ongoing research is the development of methods for sustainable afforestation and management of forests. A central role is played in this project by computer supported simulations of the dynamics of forest systems. The modeling approach adopted by CAFFE for the forest system is based on Cellular Automata (see [1]) and describes the forest as the result of competition between heterogeneous vegetable populations.

In this paper, we focus on the part of the CAFFE project that aims at designing a method to support the analysis step of software simulations of vegetable populations in the forest model. This goal is particularly relevant (and ambitious) due to the distributed modeling approach that is at the basis of the forest system simulation. Analyzing the dynamics of complex systems modeled and simulated according a distributed approach is still a challenging issue in this research field: in the simulation of the dynamics of a forest composed by different species, we can have a very complex system behavior. A very interesting, and difficult, type of simulation analysis may aim at recognizing collective emergent behaviors occurring during the system simulation. Most of the available 
approaches to analyze the dynamics of complex systems are based on statistics and probability theory and they aim at deriving macro level interpretations by aggregating and correlating variables of the micro level(s). Within the context of forest ecosystems for instance, the dynamics of the forest (e.g. biomass) is computed aggregating the features of living trees taking into account different age and dimensions of trees [2. Another common approach to complex system analysis concerns the detection and interpretation of recurring patterns [3. According to the latter approach, in this paper we propose a method to interpret detected spatial patterns that can emerge in system configuration when it is adopted a modeling approach based on Cellular Automata (CA). The originality and peculiarity of our proposal is its inspiration by Chinese Go game.

Go game, due to the simplicity of its playing rules but also to the complexity of possible configurations and the consequent complexity of playing tactics and strategies, has inspired several models (e.g. in economy, military, art, semiology, culture, and many others [4]). In Go game two populations (i.e. black and white stones) compete for survival in a territory with limited space. During the game, black and white stones situated on the Go board cannot move, but they can be put onto a board site, survive or die as result of a metaphorical competition on the local territory with neighboring stones. A key concept for the death or the survival of Go pieces is the notion of liberty: if a group of pieces has no liberties (i.e. none of its elements is adjacent to a free site), it is removed from the board.

As first step of this research, we have studied a subset of the patterns that can be observed on the Go board during the game and we have applied their interpretations to study the dynamics of a forest ecosystem modeled according to CA approach. As in Go game, in the CA-based model of forests, trees of different species live in a territory, compete for limited resources. The concept of liberty for plants can refer to favorable survival conditions and free available space for reproduction. Starting from these, and other, analogies (see Section 3), between Go rules and the behavior of entities of the $\mathrm{CA}$-based forest model, we propose to experiment the adoption of Go interpretation of Go board configurations to analyze the dynamics of the forest system.

After a summary of the CA-based model of forest previously presented in [1, in Section 3 we introduce a subset of Go patterns and we show how their interpretation can be applied to forest ecosystems. Moreover, in Section 4 we introduce an overview and some first ideas about pattern detection problem. The paper ends with future directions for the research.

\section{The CA-Based Forest Model}

In this section we give an overview of the CA-based model of forests. To introduce the model, in the following we report cell state and update rule, and the CA initial configuration.

The Cells: Each cell of the automaton represents a square portion of terrain that contains some resources (i.e. water, light, nitrogen, and potassium) and can host a tree. 
The finite set $Q$ of cell states can be defined by:

$$
Q=\left\{\mathbf{R}, \mathbf{M}, \mathbf{P}, T, \mathbf{Z}_{T}, \mathbf{N}_{T}, \mathbf{U}_{T}^{G}, \mathbf{U}_{T}^{S}, \mathbf{R}_{T}, \mathbf{M}_{T}, \mathbf{G}_{T}, \mathbf{S}\right\}
$$

where $\mathbf{R}$ is a vector referring to the amount of resources present in the cell. $\mathbf{M}$ and $\mathbf{P}$ indicate respectively the maximum amount of each resource and the amount of each resource produced by the cell at each update step. $T$ is a flag indicating whether a tree is present in the cell, while $\mathbf{Z}_{T}=\left\{z_{T}^{r}, z_{T}^{t}, z_{T}^{l}, z_{T}^{f}\right\}$ is a vector defining the size of the different parts of the tree (in our model, roots, trunk, leaves, and fruits). $\mathbf{N}_{T}, \mathbf{U}_{T}^{G}, \mathbf{U}_{T}^{S}$ and $\mathbf{R}_{T}$ are vectors related to the amount of resources needed or used by the tree living in the cell. The first one defines the amounts of each resource the tree takes from the cell. The second and the third ones define the amount of each resource needed at each update step by the tree to grow and the amount of each resource needed to survive. $\mathbf{R}_{T}$ defines the amount of each resource stored by the tree at previous update steps. $\mathbf{M}_{T}$ is a vector of threshold values for different parameters defining the tree, such as maximum size, maximum age, minimum age for reproduction, maximum number of seeds produced for each mass unity of fruits, and so on. $\mathbf{G}_{T}=\left\{g_{T}^{r}, g_{T}^{t}, g_{T}^{l}, g_{T}^{f}\right\}$ is a vector defining the growth rate of each of the parts of the tree when enough resources are available. $\mathbf{S}=\left\{s_{1}, \ldots, s_{l}\right\}$ is a vector defining the number of seeds present in the cell for each of the species growing in the territory.

The Update Rule: At each update step of the automaton, the tree present in each cell (if any) takes the resources it needs from the cell itself and uses them to survive, grow (if enough resources are available), and produce seeds. If a tree is present in cell $C(i, j)(C(i, j)$ is the cell located at position $(i, j)$ in the lattice), it takes from it a given quantity (defined by $\mathbf{N}_{T}(i, j)$ ) of each available resource $\mathbf{R}(i, j)$. The amount of resources taken depends on the size of the tree $\mathbf{Z}_{T}(i, j)$. If the resources available in the cell exceed its needs, the tree stores some resources adding them to vector $\mathbf{R}_{T}(i, j)$. Conversely, if the resources available in the cell are not sufficient, the tree uses resources stored at previous update steps. If also the resources stored are not sufficient for the tree to survive, according to $\left.\mathbf{U}_{T}^{S}(i, j)\right)$, the tree dies. The tree also dies when it reaches its maximum age defined in vector $\mathbf{M}_{T}(i, j)$. A tree can also produce some seeds, according to its age and to the size of its fruits $\left(z_{T}^{f}(i, j)\right)$, as a consequence the seed vector $\mathbf{S}(i, j)$ is updated. A newborn plant can sprout in a vacant cell, if the latter contains a seed of its specie, and again enough resources. If seeds from different species are present in the cell, the winning specie is chosen at random, with probability proportional to the number of its seeds. Moreover, we defined the update rule in order to reproduce the increasing influence that a growing tree can have on neighboring cells. For example, its roots can extend beyond the limits of the cell hosting it. Or, when it gets taller, it shades an increasingly wider area around itself, thus having a negative influence on the growth of other trees in its neighborhood. We modeled the impact of a tree in a given position on its neighborhood by making resources flow from richer cells to poorer ones. In other words, a cell hosting a large tree is poor on resources, since the tree at each update step takes most (or all) of them. If the neighboring cells are vacant, 
their resources remain unused, and thus are richer than the one hosting the tree. Therefore, if we let resources flow from richer cells to poorer neighbors, the effect is that in practice a large tree starts to collect resources also from neighboring cells.

Let $r_{h}(i, j)$ be the amount of resource $h$ contained by cell $C(i, j)$, and assume that we are using the von Neumann neighborhood. $r_{h}^{\prime}(i, j)$, the amount of resource $i$ after this update sub-step, is defined as:

$$
r_{h}^{\prime}(i, j)=\frac{r_{h}(i, j)+\frac{r_{h}(i+1, j)+r_{h}(i-1, j)+r_{h}(i, j+1)+r_{h}(i, j-1)}{4}}{2}
$$

In other words, we can see each cell as divided in four parts, each one containing the amount $r_{h}(i, j) / 4$ of resource $h$, and corresponding to one of the neighbors. The amount of resource $h$ contained in each part is balanced with the corresponding part of the neighbors. In case we adopt the Moore neighborhood, we can imagine the cells as split into eight portions.

The CA Initial Configuration: The initial configuration of the CA can be defined by the user, by setting appropriate resource parameters for each cell. Also, some trees might be already present on the territory, with all the variables defining their type. Otherwise the territory might be empty with the only presence of some seeds. According to the type of territory interested by the simulation, several types of soils and available resources for trees growth and reproduction can be set.

\section{The Go-Based Pattern Interpretation Method}

The analogies between Go game and the CA-based model of forests above sketched are going to be formalized. Very broadly in the CA-based model of forests, trees of different species live in a territory, compete for limited territory resources, can be born, die, and they cannot change their position. Moreover, the concept of liberty for plants can refer to favorable conditions for growth and reproduction. At the same time a Go board represents a territory divided in cells, and it can be viewed as a CA with three possible states for each cell: void, black or white (if there is a Go piece of the black or white team). Starting from these analogies, our proposal suggests to exploit this game to study emergent patterns in the dynamics of complex systems, by studying some spatial patterns well-known by advanced Go players [5], and to verify whether their interpretation can be suitably and fruitfully applied to interpret similar spatial patterns occurring in dynamics configurations of the CA-based forest model.

We introduce now some common spatial patterns that can emerge during Go games and that are well-known by Go players. Each spatial pattern is interpreted by Go players in terms of game competition, and we briefly describe how the interpretation of Go patterns can be applied to interpret spatial patterns that emerge from the evolution of the $\mathrm{CA}$-based model of forests. This interpretation is validated by preliminary but encouraging results of experiments conducted 


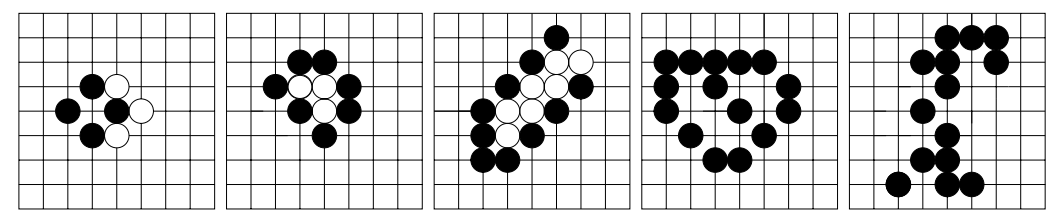

Fig. 1. Patterns in Go game: Ko, Geta, Shicho, Iki, Tsugi (from the left to the right)

on a CA-based model of the Italian Alps ecosystem (not reported here due to the lack of space but presented in [6]). An example of the Go game patterns is shown in Fig. 1].

Ko pattern: Ko is the configuration in Go game where pieces of different teams compete on a little free territory (i.e. a set of positions not occupied by stones), and no one of the two adversaries is able to take control of the territory in a stable way. In the performed simulations with the CA-based forest model, we observed similar unstable situations in which two or more tree species compete for a little territory without stable dominance. The occurrence of Ko pattern can be useful in the detection of areas in witch there are a good equilibrium between the living species.

Geta pattern: Geta pattern corresponds to the local capture of a group of adversary pieces by a set of stones that surrounds it. Similarly if a group of trees is surrounded by plants of another specie and it is forced to be limited within a little and close territory zone, sooner or later it will die. In fact, trees and plants that are forced in a little zone have little space to harvest the needed resources for survival and reproduction. The occurrence of Geta pattern can be a good sign of the presence of a dominant specie in the given area.

Shicho pattern: Shicho is a pattern in which a group of Go stones expands itself towards another side of the Go board. Shicho does not imply the movement of single stones that are part of the moving group. Group movement occurs in timespace (i.e. group movement is the result of stones removal from some positions and the positioning of others in adjacent ones). In forests Shicho occurs when an homogeneous group of plants is situated in a zone where resources are not suitable to satisfy their needs. Since new born trees are more likely to survive if they grow up on areas rich of resources, also in this context, we can observe as emergent phenomenon, a group shifting in space-time. The occurrence of Shicho pattern may indicate a soil impoverishment or the rising up of a new dominant specie in the area.

Iki pattern: Iki is a strong pattern that can not be captured by the adversary. Iki corresponds to a part of Go board surrounded by stones of the same color, with some free positions in its inner side to form two 'eyes', in Go jargon. This formation has two internal liberties that, according to Go playing rules, cannot be occupied by adversary pieces. A spatial pattern in forest dynamics similar to Iki is characterized by a strong group that can survive for long time assuring part of the territory to its individuals. In fact, trees of this group have vacant cells in 
their neighborhood and this guarantees the availability of space and resources for their survival and reproduction. The occurrence of Iki pattern shows the stability of the involved specie in the given area.

The Tsugi pattern: Tsugi is a Japanese word that means 'connection'. Connections are very important in Go competition, because two stones connected to form a group are stronger than they alone. In fact, it is more difficult for the adversary to build a group able to surround connected stones (i.e. it may require a lot of stones). Tsugi phenomenon occurs in a natural way also in forests. Each group of plants expands itself by reproduction, and when two groups expand toward one another, there is the possibility to create a connection between them. As in Go game, two connected groups of plants are stronger because they can support each other. When a plant dies neighboring ones can replace it, and when two groups are connected, neighbors increase in number. Beside this aspect, an isolated little group of trees can easily fall in the Geta phenomenon but if it is connected to another group, it is no more possible to surround it. Tsugi pattern occurs in particular in a situation of a new territory colonization or during a repopulation phase of a given area. In the both cases it indicates an unstable situation.

All these patterns can be used for a meaningful interpretation of important phenomena in some simulation scenarios. In particular they can be useful, for example, in the case of artificial repopulation of forest in a given area with the introduction of new species. In this case it is important to understand if the new specie can survive and what are the reactions of the other living species. But while the occurrence of Ko phenomenon means a good equilibrium between species, the frequency of Iki and Tsugi indicates the formation of a strong dominance presence in the area, and Geta and Shicho mean the disadvantage of a specie in comparison with the others. All these considerations can support the decision maker in the illustrated domain problem.

A central issue to be considered in developing a method for pattern interpretation concerns the development of a method to support the detection of emergent patterns during system dynamics. This task can be very difficult for a human operator that analyzes the dynamics of the CA-based forest model, in particular for large scenarios involving several tree species. Therefore the main future work that starts from first positive results on Go-based pattern interpretation concerns the development of a detection method capable of recognizing known patterns. In the following section we report an overview and some first ideas about pattern detection problem.

\section{Towards a Pattern Detection Method}

In its most general sense, pattern detection can be defined as "the extraction of consistent information from (possibly noisy) spatiotemporal data". Alternatively, it can be defined as "the act of taking in raw data and taking an action based on the category of the data" 7]. As such, it can be accomplished by a large set of many different machine learning methods. Pattern recognition aims to classify 
data (patterns) based on either a priori knowledge (supervised learning) or on statistical information extracted from the patterns (unsupervised learning). In general, a complete pattern recognition system consists of (1) a sensor that gathers the observations to be classified or described; 2 ) a feature extraction mechanism that computes numeric or symbolic information from the observations; (3) a classification or description scheme that does the actual job of classifying or describing observations, relying on the extracted features. In our applications, the observations are already given by the structure of the cellular grid at a given time step. Thus, step (1) is not necessary. Many Artificial Intelligence and statistical techniques have been developed for feature extraction (point (2)). Some examples are: discriminant analysis, principal component analysis, principal curve, factor analysis, independent component analysis, some forms of clustering and feature selection. All these techniques may be useful for our application, particularly in presence of large cellular grids. We are particularly interested in discriminant analysis, where new basic features may be considered as the current states of the grid cells and new feature may be constructed (eventually replacing the old ones) via linear or non-linear combinations, so that classification (point (3)) is easier. The choice of the classification scheme (point (3)) for pattern detection in our application is probably the most delicate: a large number of machine learning techniques have been used with success in many pattern detection applications which have similarities with ours. For example, in [8], Ripley introduces the use of many different Neural Networks models for pattern detection in many applications, such as classifying galaxies by shape, identifying fingerprints, highlighting potential tumors on a mammogram, handwriting recognition. While the second, third and fourth of these applications can be considered "simpler" than ours, the first one has some similar characteristics, in particular for the fact that galaxies are modelled as complex systems, in a similar way as we do for forests. In [9], Babuskame presented a way to solve some pattern recognition applications by means of fuzzy systems. Fuzzy rules may provide more flexibility and decisional power to the system. In [10] contains many contributions on the use of evolutionary techniques (like Genetic Algorithms) for solving many pattern detection applications. The contribution given by Wah et al. in this book (chapter 5) is particularly interesting, because it deals with the generalization of learned rules in genetic-based learning. Furthermore, chapters 9 to 13 of this book present some hybrid techniques integrating Fuzzy, Neural and Genetic approach. Given the complexity of our application, we are particularly interested in this last kind of system. Fuzzy, Neural and Genetic systems, in fact, are characterized by a high degree of diversity. Hybrid pattern recognition schemes have been proposed in order to combine their strengths and avoid their weaknesses. Some good discussions about this issue are contained in [11, where several techniques are combined into single pattern recognition systems. Hybrid methods have been known about for a long time, but they have gained new interest only recently. Nevertheless, in our opinion, they have never been applied to complex systems like detection of spatial patterns in vegetable population and forest dynamics. 
This research field looks promising and interesting and we plan to investigate it in the future.

\section{Conclusions and Future Works}

In this paper we have proposed a method for pattern interpretation of emergent phenomena that aims at supporting ecosystem management in the study of forest systems by simulation. This proposal has been validated by preliminary but encouraging results of experiments conducted on the CA-based model applied to an area of Italian Alps ecosystem. These experiments were conducted to verify the correct evolution of Go-like patterns with reference to the forest simulation scenario (their positive results are available in [6]). A deeper experimentation campaign is under development in collaboration with ARC's domain experts in order to apply the presented approach on real data scenarios.

The main future work that starts from these results concerns the development of a detection method capable of recognizing and interpreting known patterns during system dynamics (first ideas have been introduced in Section 4). Another important future development of this research can be the study of new patterns for the interpretation of other phenomena obtained by the introduction of other system elements in the model (e.g. urbanization or pollution as effect of the human presence, or desertification as effect of other natural interactions).

\section{References}

1. Bandini, S., Pavesi, G.: "Simulation of vegetable populations dynamics based on cellular automata" In Bandini, S., Chopard, B., Tomassini, M., eds.: Cellular Automata, Volume 2493 of LNCS, Berlin, Springer-Verlag (2002)

2. D.G.Green, "Modelling plants in landscape", in Plants to Ecosystem - Harek T. Michalewicz, ed. CSIRO, Lollingwood Ans., 1997.

3. S.Wolfram, "Cellular automata as models of complexity", Nature, 311:419-424, 1984.

4. Reysset, P.: Le Go: aux sources de l'avenir. Chiron (1994)

5. Soletti, G.: Note di Go. FIGG (Federazione Italiana Giuoco Go). Avaiable for download at www.figg.org

6. Bandini, S., Manzoni, S., Redaelli, S.: "Toward the Interpretation of Emergent Spatial Patterns through GO Game: The Case of Forest Population Dynamics" In proceedings of Simulation and Formal Analysis of Complex Systems (WOA 2005)

7. R. O. Duda, P. E. Hart and D. G. Stork. Pattern classification (second edition). Wiley. New York. 2001.

8. B. D. Ripley Pattern Recognition and Neural Networks. Cambridge University Press. 1996.

9. R. Babuska. "Fuzzy clustering algorithms with applications to rule extraction". In P.S. Szczepaniak and P.J.G. Lisboa, editors, Fuzzy Systems in Medicine, pages 139-173. Springer-Verlag, Heidelberg, 2000.

10. S. K. Pal and P. P. Wang Genetic Algorithms and Pattern Recognition. Boca Raton, FL: CRC Press. 1996.

11. H. Bunke and A. Kandel. Hybrid methods in pattern recognition. World Scientific Series in Machine Perception and Artificial Intelligence, Vol. 47. 2002. 